\title{
A Rotational Speed Measurement System Based on STC89C52 Single Chip Microcomputer for Micro-turbojet Engine
}

\author{
Zekun Liu, Zhou Yan, Jianhui Xu, Jingbo Guo, Fengyu Wu, and Cong Ding \\ School of Engineering Training Center, Shenyang Aerospace University, Shenyang 110136, China.
}

Keywords: rotational speed measurement, hall sensor, SCM, micro-turbojet engine.

\begin{abstract}
In order to improve the rotate-speed precision of micro-turbojet engine and reduce costs of measuring equipment, a rotational speed measurement system based on STC89C52 and using Hall Sensor was researched. A simulative working process, based on the model of micro-turbojet engine and using DC motor to drive spindle, was studied. A relatively better result was gotten by using this system and equipment. Compared with other methods, the circuits is simpler with higher precision and stronger capability of resisting disturbance, especially when the speed range is great.
\end{abstract}

\section{Introduction}

Nowadays, people know more and more about the model aircraft, especially those equipped with micro-vortex jets attract more and more people. The key to the thrust control of the turbojet engine is to control the rotation speed of the shaft, so the engine speed is accurate and timely. Measurements are very important. In order to meet the needs of amateurs for vortex modelling, the commodity machines on the market are more expensive for general enthusiasts. Based on the M/T measurement method, this paper uses the Hall effect to develop the design. Using the STC89C52 microcontroller as the development carrier, the circuit is simulated in the proteus software. Finally, the board is manually soldered and the system is tested for its feasibility. This system uses common components on the market, greatly reduces the production c ost on the basis of satisfying the measurement requirements, and is suitable for ordinary enthusiasts.

\section{Overall Plan}

Due to the large rotating speed range of the micro turbojet engine, the machining accuracy of the engine shaft is required to be high. Therefore, the $\mathrm{M} / \mathrm{T}$ method is used to measure the rotation speed of the shaft. When the speed is measured, the speed period is changed according to the actual rotation speed. When the rotation speed is low, the measurement period is long, and as the rotation speed increases, the measurement period becomes shorter, which improves the real-time and accuracy of the rotation speed measurement. At the same time, in order to reduce the difficulty of machining the shaft, the magnetized nut is used to fix the gas pressure wheel, and the Hall switching element is used to convert the measured magnetic field change into a voltage change. Compared with using the infrared measuring speed, it is not necessary to open a hole in the nut and reduce The processing difficulty.

\section{Design of Speed Measurement System}

\subsection{Speed principle.}

According to the principle of speed measurement can be divided into three categories. The first type is the frequency measurement method ( $\mathrm{M}$ method), that is, counting the number of pulses generated due to rotation of the shaft within a determined time, the calculation can obtain the rotation speed; the second type is the measurement method (T method), which measures the interval time between pulses, and then calculate the speed; the third is the frequency measurement method $(\mathrm{M} / \mathrm{T}$ 
method), on the one hand to count the pulse sent by the sensor, on the other hand, count the pulse measurement time, and then calculate the speed. The $\mathrm{M}$ method has high measurement accuracy when the rotation speed is high, and the T method has high accuracy when the rotation speed is low, and the $\mathrm{M} / \mathrm{T}$ method can take both the measurement accuracy of the high and low rotation speeds into consideration and set different sampling times at different rotation speeds. Further improve the measurement accuracy.

Non-contact measurement speed measurement principle has photoelectric effect principle and Hall effect principle, because the photoelectric effect principle needs to open holes on the lock nut, increasing the machining difficulty, so the Hall effect principle, the lock nut is magnetized, Reduces the difficulty of processing at the same time is conducive to the dynamic balance of the shaft.

\subsection{Programming.}

Start, speed and stop the starter motor using the microcontroller. After the MCU is powered on, wait for the command to start the motor and measure the speed. The M/T method is used to measure the speed. The default sampling time of the speed measurement system needs to be determined in advance for the first measurement. The default speed measurement period is $1 \mathrm{~s}$, in order to further improve the measurement accuracy. As the speed decreases, the sampling time needs to be extended appropriately, and as many pulses as possible are measured to reduce the measurement error. Therefore, a series of sampling times are set corresponding to different speed ranges, and the measured first speed and the predetermined speed are determined. The interval is compared to obtain the next sampling time for the next rotation speed measurement. The program flow chart is as follows:

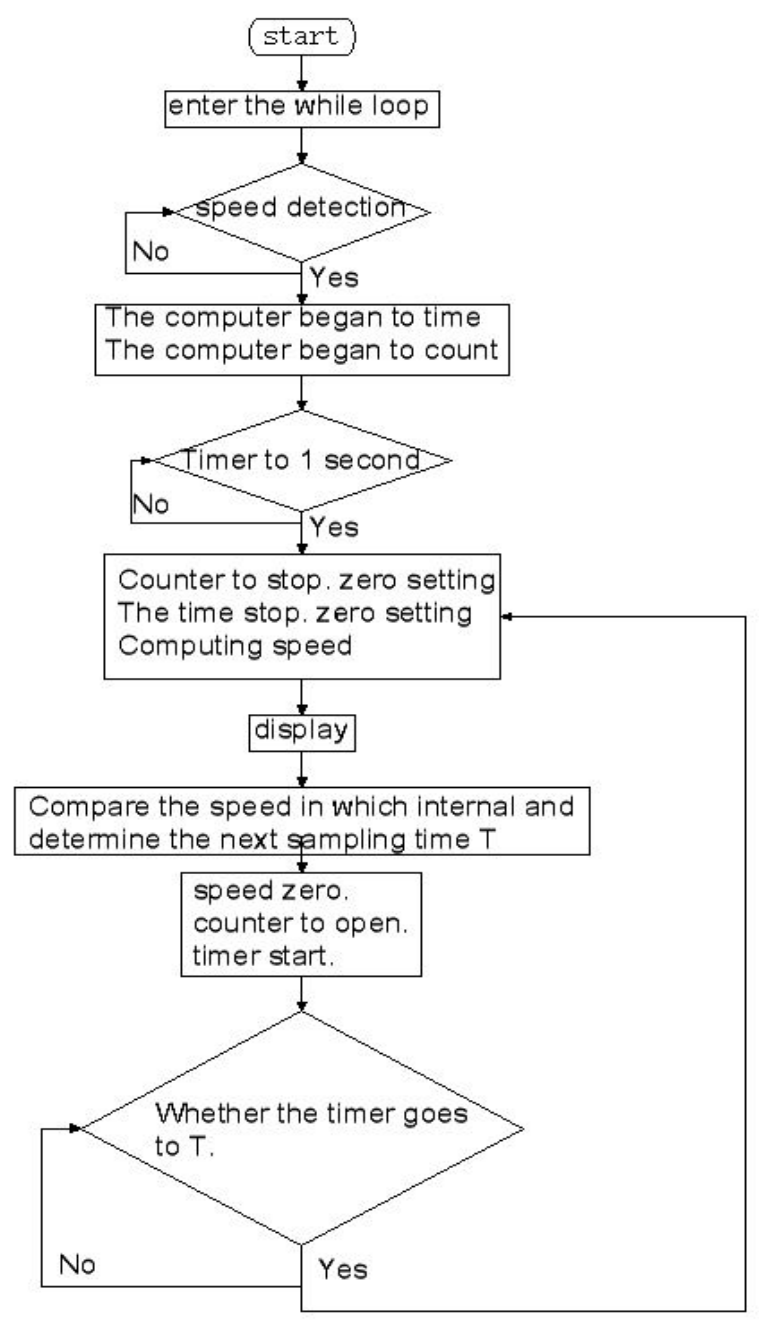

Fig. 1 Program flow chart 


\subsection{System design.}

The system can control the start and stop of the starter motor, and the start and stop of the speed measurement will display the measured speed. The start and stop of the starter motor and speed measurement can be controlled using two buttons, and the microcontroller detects whether the corresponding button is pressed. Since the motor has a large operating current, a drive circuit is required for driving. The measurement of the speed requires two counters to count the number of external pulses and internal crystal pulses, a timer to determine the sampling time, and a Hall sensor to generate pulses following the axis. After the speed calculation is completed, the speed needs to be displayed to facilitate the calculation of the speed error.

\subsection{Circuit design}

\subsubsection{Minimum system module}

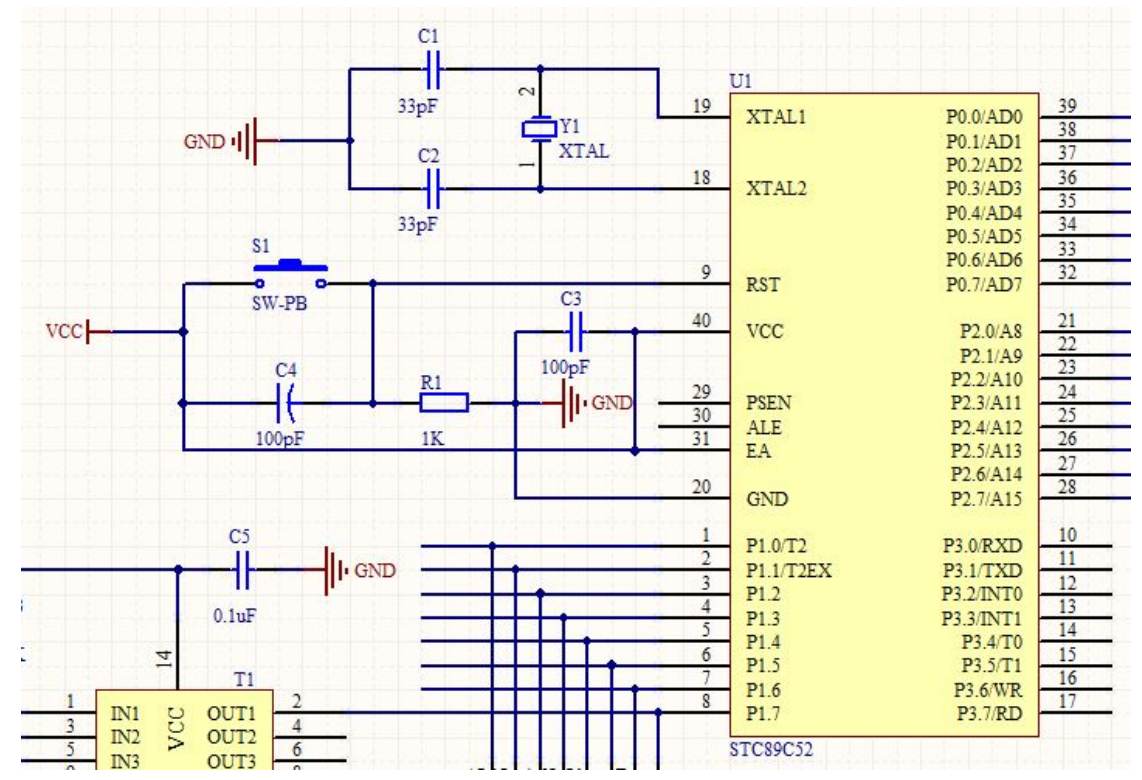

Fig. 2 Crystal circuit and reset

\subsubsection{Motor drive module}

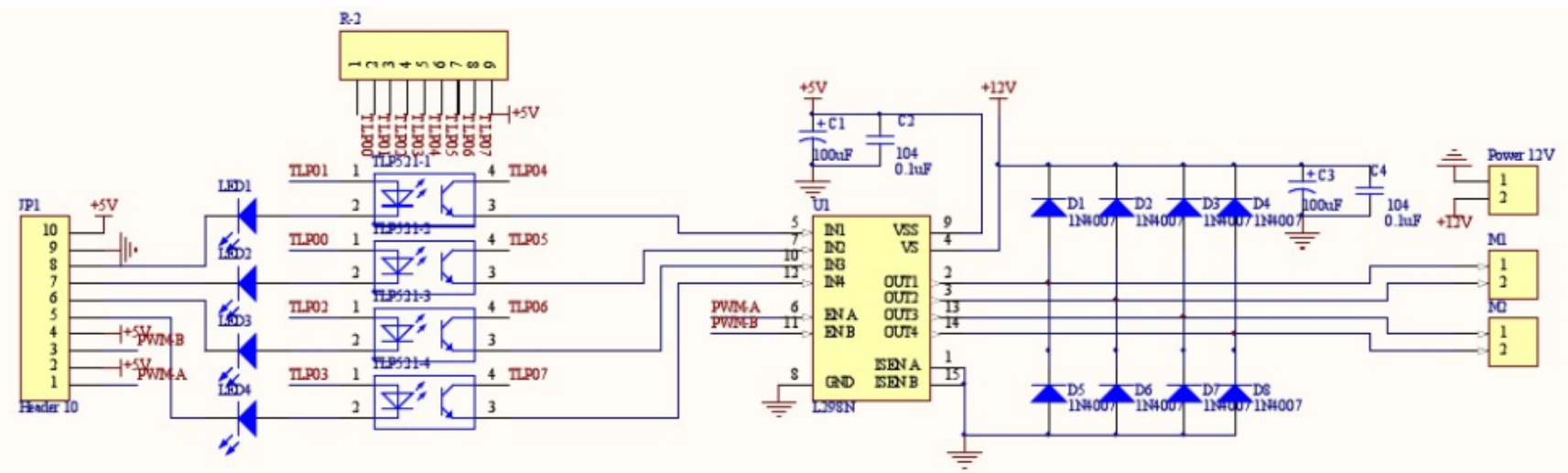

Fig. 3 Motor drive circuit 


\subsubsection{Speed measurement module}

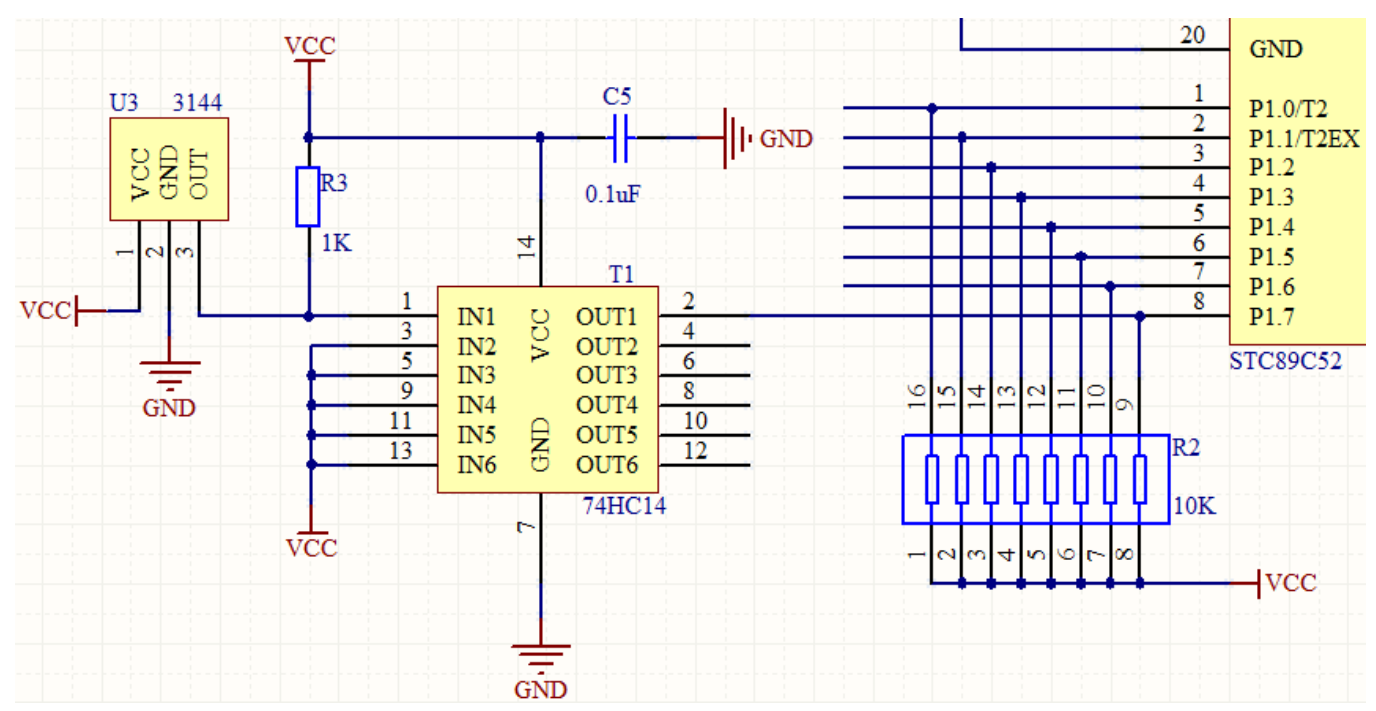

Fig. 4 Measuring rotate speed circuit

\subsubsection{Speed display module}

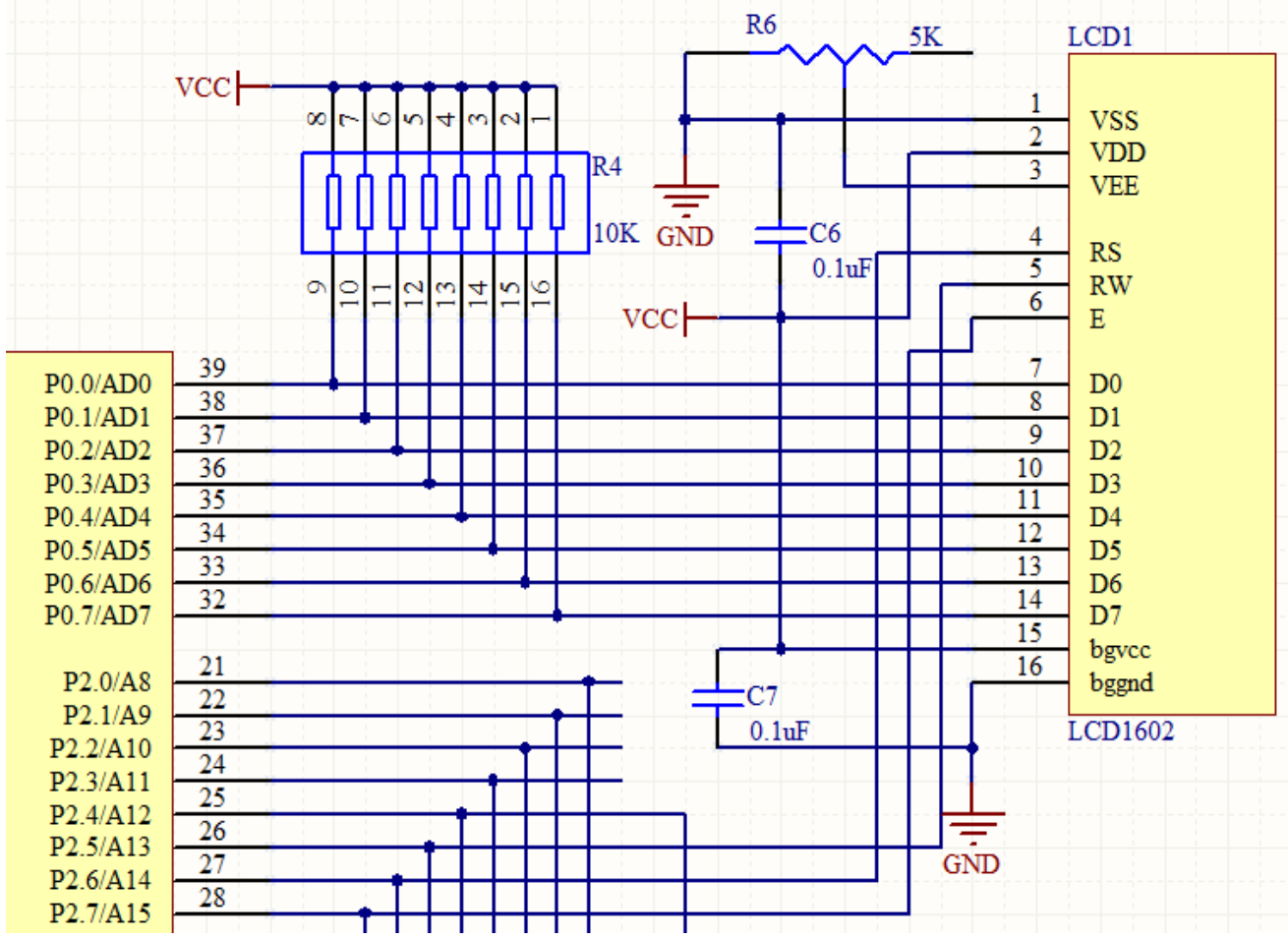

Fig. 5 LCD display circuit

\section{Simulation of Speed Measurement System}

\subsection{Basic method.}

In order to verify the feasibility of the system, we found problems that may be encountered in the fabrication of the device. Before proceeding with the experiment, use Proteus for simulation. According to the circuit designed above, set up the entire system in the simulation software and set up an external pulse generation source instead of measurement. At the time of the rotation speed pulse, set the frequency of the pulse generation source in the software, use the developed system to measure the frequency of this pulse, calculate the corresponding rotation speed and display it on the LCD 
screen, and then compare it with our set value to determine the feasibility of the program.

\subsection{Simulation Results}

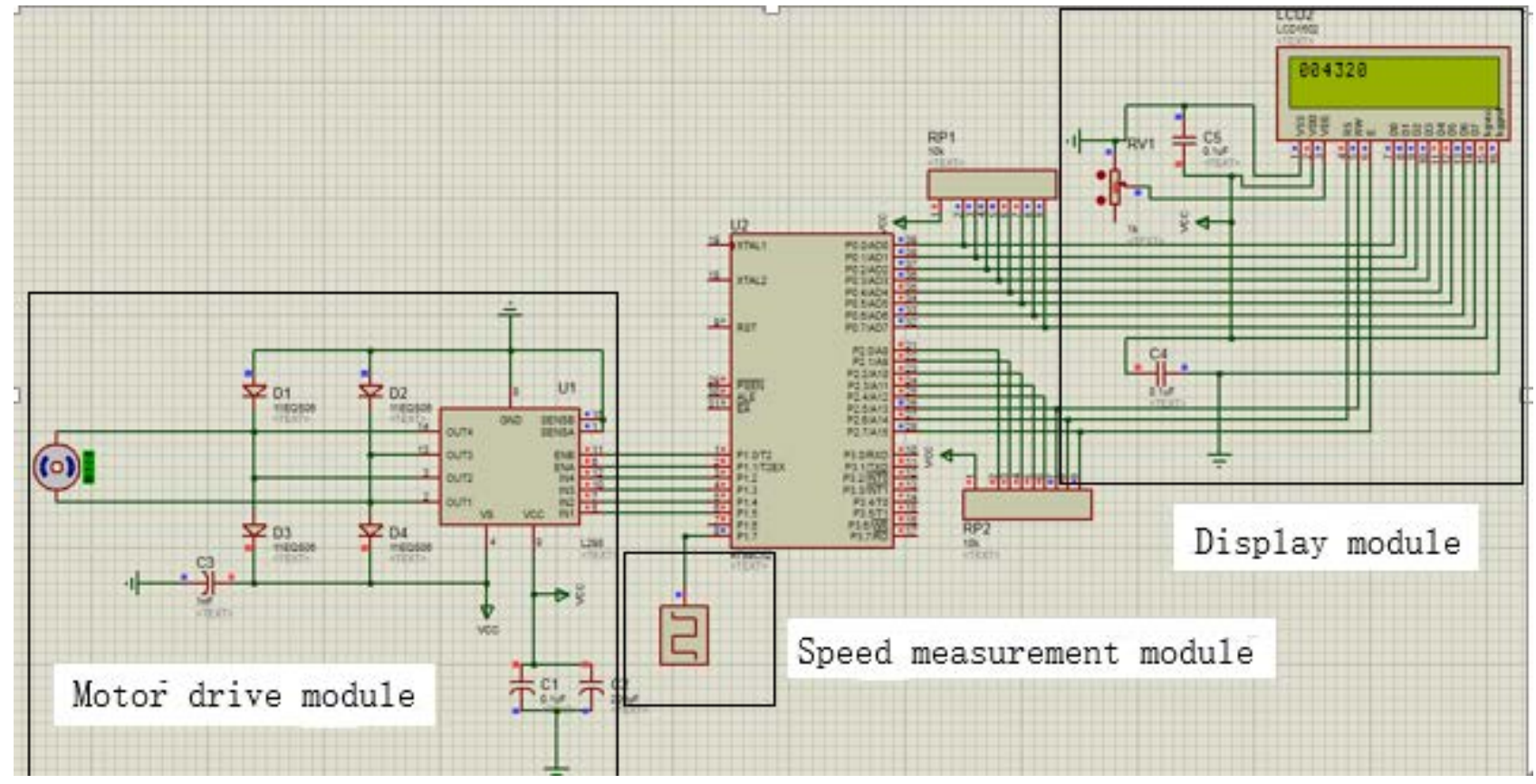

Fig. 6 Result of simulation

\section{Conclusion}

In this paper, the $\mathrm{M} / \mathrm{T}$ speed measurement method of micro turbojet engine based on single chip microcomputer is proposed. It can be seen from the speed measurement process that the system can measure the rotor speed quickly and accurately, which reduces the machining requirements of mechanical components compared with other methods. Different sampling speeds use different sampling times to improve the measurement accuracy at high and low speeds, which is beneficial to engine control. This method is applicable not only to the rotation speed measurement of the engine rotor, but also to the rotation speed measurement of other shaft parts, such as the wind turbine rotor. However, due to the limited operating speed and memory of the selected microcontroller, the real-time measurement cannot be performed for the high rotation speed. Therefore, the error will increase when the high speed is measured.

\section{Acknowledgements}

Fund project: Shenyang Aerospace University Student's Platform for Innovation and Entrepren-eurship Training Program.

First author: Zekun Liu (1996 -- ), male, undergraduate, main research direction is aircraft design.

Corresponding author: Zhou Yan (1982 -- ), male, undergraduate, main research direction is the application of numerical control technology and mechanical design research.

\section{References}

[1] Z.P. Wang: Speed Measurement Design Based on 89 C51 (MS., Southeast University, China 2005), p.8-30. (In Chinese)

[2] L. Liu, X. Wang: Machinery, Vol. 34 (2007) No.3, p.58-60. (In Chinese)

[3] S.L. Zhao, J.H. Xie and Y. F. Liu: Jiangsu Electrical Apparatus, Vol. 28 (2008) No.10, p.53-56. (In Chinese) 
[4] Z.Q. Ding: Journal of Agricultural Mechanization Research, Vol. 32 (2010) No.5, p.81-83. (In Chinese)

[5] J. Niu, J. Zhou and N. Gou: Electronic Test, Vol. 15 (2008) No.6, p.75-78. (In Chinese)

[6] J.H. Gu: Popular Science and Technology, Vol. 10 (2007) No.11, p.112-113. (In Chinese) 